\title{
Biliary cystadenoma in an endemic zone of hydatid cyst: A rare surgical surprise
}

\author{
Krishna Ramavath ${ }^{1}$, Lileswar Kaman ${ }^{1}$, Ashish Gupta ${ }^{1}$, Abhinav Singh ${ }^{1}$, and Asim Das ${ }^{2}$ \\ Departments of ${ }^{1}$ General Surgery and ${ }^{2}$ Histopathology, \\ Post Graduate Institute of Medical Education and Research, Chandigarh, India
}

\begin{abstract}
The advancement of radiological investigations has led to the early and incidental detection of hepatic cystic lesions. These are most commonly the simple cysts but can be malignant as well. Despite the recent advances, these lesions still pose a diagnostic as well as therapeutic challenge. The biliary cystadenomas and carcinomas form around $5 \%$ of all the malignant cystic lesions of liver. These lesions are hardly diagnosed preoperatively and are usually a histopathological surprise. They warrant a surgical excision. Herewith, the authors are describing a case of cystic hepatic neoplasm initially misdiagnosed as hydatid cyst of liver and discovered to be a vascular cystic lesion intraoperatively. This patient underwent resection of the lesion and was discovered to harbour biliary cystadenoma on histopathological specimen. (Ann Hepatobiliary Pancreat Surg 2020;24:85-89)
\end{abstract}

Key Words: Biliary cystadenoma; Cystadenocarcinoma; Hydatid cyst of liver

\section{INTRODUCTION}

Hepatic cystic lesions (HCL) are increasingly been encountered in modern day surgical practice. ${ }^{1}$ These are usually asymptomatic and are incidentally found on radiological investigation for unrelated problem. The lesions are usually benign however can be malignant as well. ${ }^{2,3}$ These cystic lesions comprise of constellation from a benign simple cyst to a malignant cystadenocarcinoma. The benign lesions can be observed but the malignant ones need to be investigated and treated properly.

Biliary cystadenoma $(\mathrm{BC})$ are rare HCL. They comprise of $5 \%$ of the cystic lesions. ${ }^{4}$ These lesions are more commonly reported in middle aged group females. ${ }^{5}$ They are usually asymptomatic however can lead to symptoms when the adjacent visceral structures are compromised. ${ }^{6}$ Echinococcal cysts or hydatid cysts of the liver can closely mimic these lesions in the endemic areas. ${ }^{7,8}$ The diagnosis of hydatid cyst is essentially radiological according to the World health organization (WHO) classification. The false negative serology in hydatid cyst also poses a diagnostic dilemma. ${ }^{9}$ Herewith the authors are describing a male patient with large HCL involving the right lobe of the liver. He was diagnosed with hydatid cyst on initial radiological tests and subsequently found to have vascular cystic neoplasm intraoperatively. He underwent an excision of the lesion and discovered to harbour a biliary cystadenoma without a focus of malignancy. The rarity of these lesions in males along with the associated diagnostic and surgical surprise makes this case a unique report.

\section{CASE}

A 25 year male without any associated comorbidities presented to our outpatient department with complaints of pain in right hypochondrium for last 5 years which was intermittent and dull aching in nature. There were no aggravating or relieving factors. There were no associated symptoms. Clinically there was a large mass involving the right hypochondrium and lumbar regions. This was mobile with respiration and had irregular surface and margin. The dullness over the mass was continuous with the liver

Received: October 5, 2019; Revised: December 2, 2019; Accepted: January 19, 2020

Corresponding author: Lileswar Kaman

Department of General Surgery, Post Graduate Institute of Medical Education and Research, Sector 12, Chandigarh 160012, India Tel: +91-9878254574, Fax: +91-172-2744401, E-mail: kamanlil@yahoo.com

Copyright (C) 2020 by The Korean Association of Hepato-Biliary-Pancreatic Surgery

This is an Open Access article distributed under the terms of the Creative Commons Attribution Non-Commercial License (http://creativecommons.org/ licenses/by-nc/4.0) which permits unrestricted non-commercial use, distribution, and reproduction in any medium, provided the original work is properly cited. Annals of Hepato-Biliary-Pancreatic Surgery • pISSN: 2508-5778 • elSSN: 2508-5859 
dullness. The provisional diagnosis of a hepatic mass was made and subsequent haematological and radiological investigations were ordered. His renal functions, liver functions and complete blood counts were within normal range. Ultrasonography of the abdomen revealed two exophytic hypoechoic lesions with multiple septae arising from right lobe of liver. There was no Intrahepatic biliary radical dilatation. Portal vein was normal. Gallbladder was distended and normal. There was presence of hydronephrosis in the right kidney and other viscera were normal. Amoebic serology and hydatid serology were negative. The tumor markers Alpha fetoprotein $(1.02 \mathrm{ng} / \mathrm{ml})$, carbohydrate antigen 19.9 (34 IU/dl) and Carcinoembryonic antigen (1.06 $\mathrm{ng} / \mathrm{ml}$ ) were within normal range.

He underwent a Contrast enhanced computerized tomography (CECT) abdomen which revealed an exophytic multiloculated hypodense lesion ( 9 to $50 \mathrm{HU}$ ) with with multiple thick septae seen in segment IV a, IV b and V. There was splaying of right portal vein and left portal vein with mild attenuation of right portal vein calibre. Main portal vein and hepatic veins were normal. The lesion was abutting the anterior branch of right hepatic artery. There was no intrahepatic biliary radicle dilatation. Common bile duct was prominent. The lesion was abutting the abdominal wall anteriorly, the psoas muscle and IVC medially and it was displacing few small bowel loops. It was also abutting the antropyloric region of stomach and duodenum and displacing head of pancreas. The lesion was compressing the right mid ureter with upstream dilation. Tiny calcific foci seen within the cyst. Rest of the visualised abdominal organs were normal (Fig. 1).
With the preoperative diagnosis of hydatid cyst this patient was optimized and prepared for surgery. He underwent the DJ stenting of the right ureter followed by laparotomy with excision of the cystic mass. Intraoperatively, a large mass involving the right lobe of liver was identified. This mass was abutting the antropyloric region of the stomach and right kidney but could be separated from them. Fluid aspiration from the mass yielded mucinous fluid hence a decision of formal excision was made. The mass was excised without any capsular breach. The cut section of mass showed multiloculated cystic lesion with mucinous fluid within it (Fig. 2). The patient had an

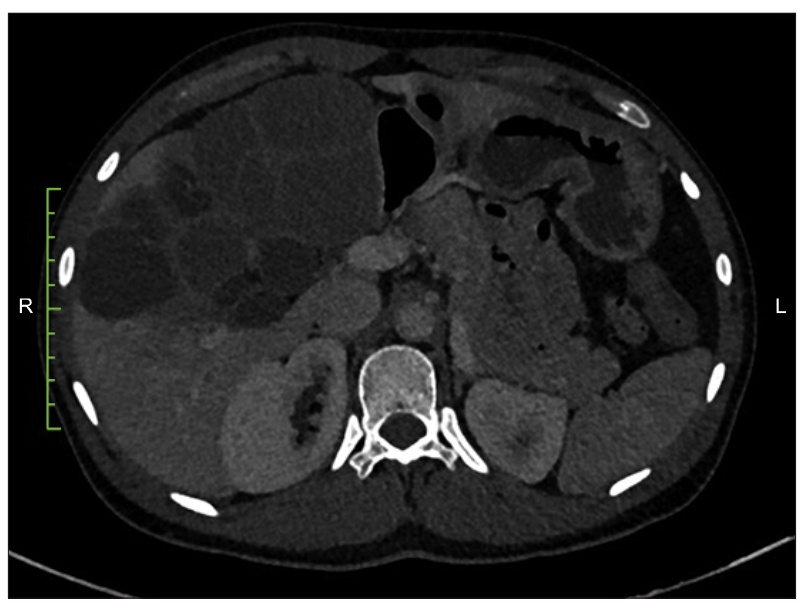

Fig. 1. Multiloculated hypodense lesion ( 9 to $50 \mathrm{HU}$ ) with with multiple thick septae seen in segment IV A, IV B and $\mathrm{V}$. The lesion is abutting the anterior abdominal wall anteriorly, medially it is abutting the psoas muscle and IVC and displacing few small bowel loops. It is also abutting the antropyloric region of stomach and duodenum and also displacing head of pancreas.
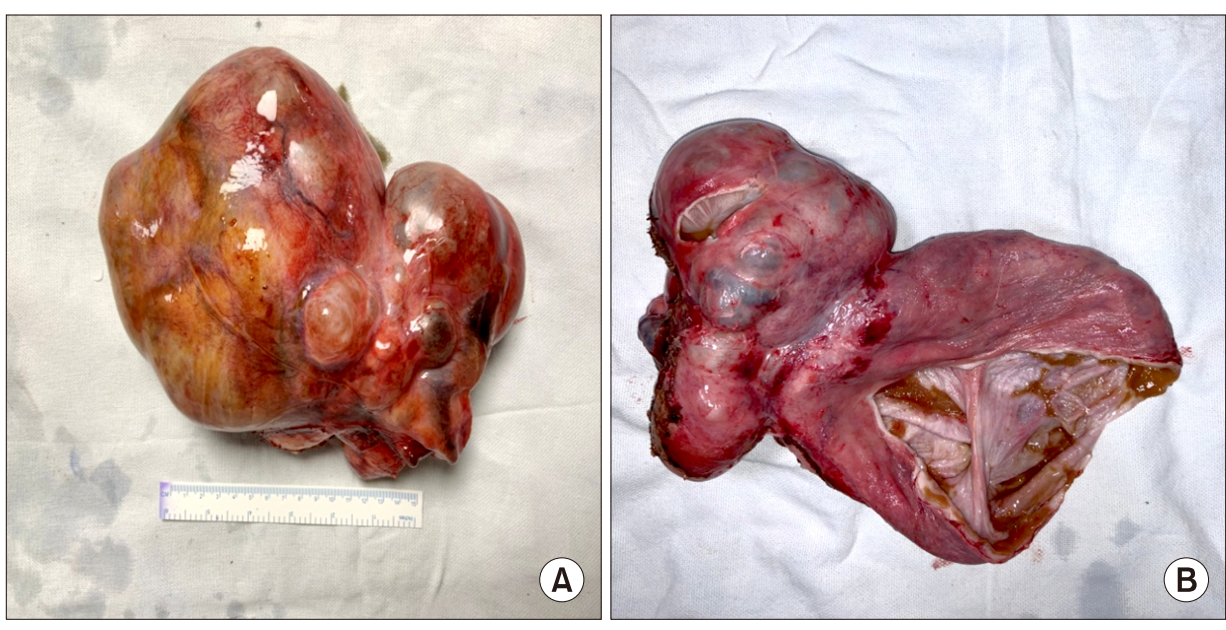

Fig. 2. (A) Resected gross specimen of $\sim 18 \times 16 \times 9 \mathrm{~cm}$ with bosselated surface containing multiple cysts. (B) Cut section of whole specimen containing multiple cystic lesions. Luminal aspect of the cyst was smooth and contains mucinous fluid. 

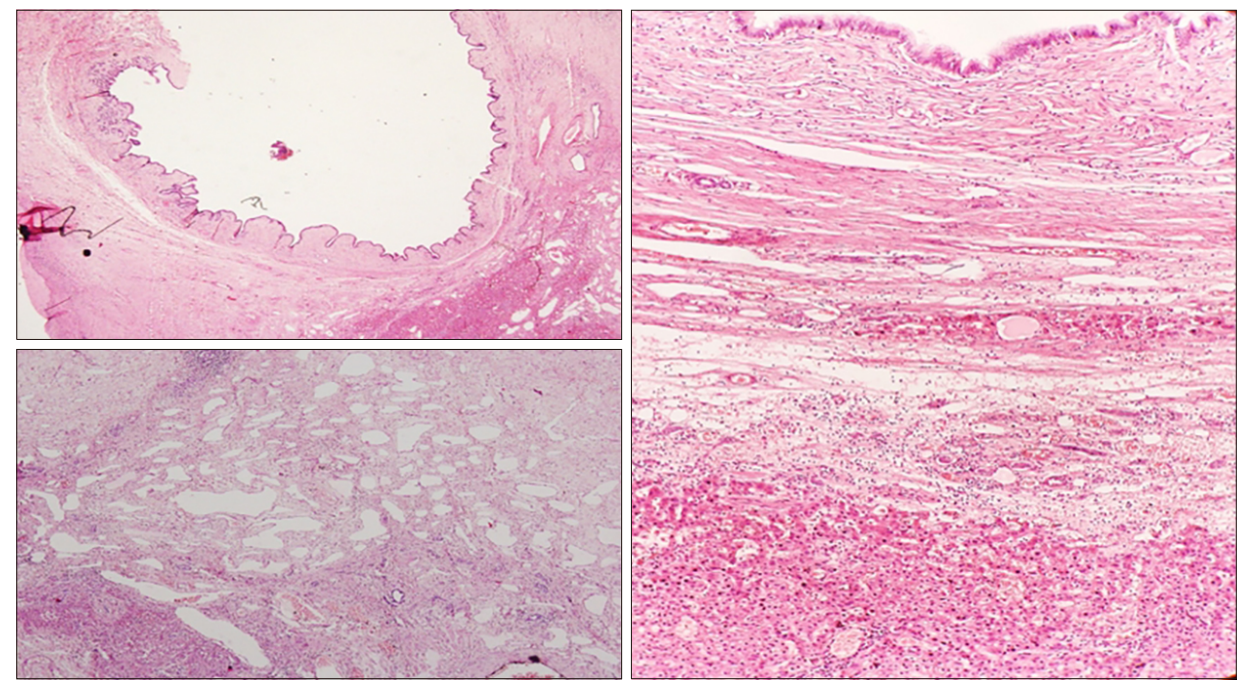

Fig. 3. Histopathology report: multiloculated cyst contains variable size of cystic spaces lined by mucinous epithelium, at places it is replaced by macrophages sheets. It contains fibrous septae, fibrocollagenous tissue and moderate degree of lymphocytes rich chronic inflammation with lymphoid aggregates. Many proliferating vascular and lymphatic channels are also seen. There was no evidence of ovarian stroma. No neoplastic cells were seen. Overall features of biliary cyst adenoma without ovarian stroma.

expectant postoperative course and was discharged on sixth post operative day without any associated complications.

The gross specimen on histopathological examination revealed multilocular cyst with varying size septae. The luminal side of the cyst was smooth and lined with mucin. On microscopic examination, there was presence of multiloculated cyst lined by single layer of mucinous epithelium. The cyst wall contained fibrocollagenous tissue with macrophages and lymphocytes at places. There was the presence of proliferating lymphatic and vascular channels. There was absence of ovarian stroma, parasite or a neoplastic process. Estrogen and Progesterone immunostains were negative. The features were consistent with a biliary cystadenoma without any focus of malignancy (Fig. 3).

\section{DISCUSSION}

HCL are increasingly been encountered in the modern day surgical practice. ${ }^{1}$ They include the spectrum of benign simple cysts to a malignant cystic lesion. The parasitic infections also add to the surgical challenges especially in the endemic areas. ${ }^{7,8}$ These lesions are often benign but can be malignant as well. They are usually indolent and are diagnosed on radiology for an unrelated pathology. ${ }^{1}$ The wide spectrum of biochemical and radio- logical investigations along with the fluid aspirate parameters can precisely lead a definitive diagnosis in difficult cases. However the index case was a challenging one as the diagnosis was confirmed on postoperative histopathological examination.

Biliary cystadenomas form $5 \%$ of the total HCL. ${ }^{4}$ The incidence rate of these lesions is 1 in 20000- 1 in $40000 .^{10}$ The etiology of these lesions is largely unknown however a congenital malformation of biliary epithelium is suggested. $^{5}$ These are indolent lesions and the rate of growth is very slow. They can turn malignant if neglected. The middle aged females form $85 \%$ of the patients. ${ }^{11}$ Liver $(85 \%)$ is the most commonly involved viscera however, extrahepatic bile ducts as well as gallbladder can also be affected in rare cases. ${ }^{12}$ The presence of this cyst in a male patient and mimicking a hydatid cyst in an area endemic for echinococcosis makes the index patient an unique report.

Biliary cystadenomas are usually asymptomatic. These are indolent and rarely symptomatic. These symptoms arise secondary to the the adjacent visceral compromise. There may be obstructive jaundice due to common bile duct compression, ${ }^{13}$ bilateral pedal edema or deep vein thrombosis in case of inferior vena caval obstruction. ${ }^{6}$ The present patient had hydronephrosis secondary to ureteral compression necessitating a preoperative ureteral stenting. 
The wide variety of HCLs in the liver pose a diagnostic challenge. The hydatid cyst of the liver as well as the liver abscess are the most common space occupying lesions (SOL) that resemble cystadenomas. ${ }^{7,8}$ The aspiration of the liver abscess is therapeutic but it is contraindicated both in hydatid cyst as well as cystadenoma ${ }^{14}$ due to risk of anaphylaxis as well as seeding respectively. These cysts may be a differential diagnosis for intrahepatic cholangiocarcinoma or the cystic variant of cholangiocarcinoma as well. ${ }^{6}$ Vonmeyenburg complex and other variants of hepatic plate dysgenesis syndromes may closely mimic these lesions. ${ }^{15}$

The BC is usually an incidental finding. The liver function test and biochemical profile is usually normal. The serum tumor markers are recommended to rule out any hepatic malignancy. Serum hydatid serology and amoebic serology must be performed in endemic areas. ${ }^{6}$ The false negative rates of the serologic tests are in range of $20 \%{ }^{9}$ which means the hydatid cyst being the more common SOL in endemic area should be considered in priority. The present case is the classical example where the presence of mutilocular cyst along with the endemicity of hydatid prompted the surgeons as well as the radiologists towards this diagnosis.

The ultrasonography shows a round or oval shape, irregular border, hypoechogenic echo pattern, wall enhancement and dorsal shadowing due to calcified areas. CECT abdomen shows thickened wall with papillary projections, septations and calcifications. ${ }^{4}$ The presence of thick septations along with infiltration signifies malignant transformation to a carcinoma. The differentiation of malignancy on radiology is difficult even with the recent advancement of diffusion weighted magnetic resonance imaging. ${ }^{16}$ Contrast enhanced ultrasound can differentiate cystadenoma from a cystadenocarcinoma when other radiological modalities fail to do so. ${ }^{1}$ Positron emission tomography (PET) with high fluorine-18-2-fluro-2-deoxy-D-glucose (FDG) uptake may also help in delineating malignant lesions. ${ }^{17}$ The surgical excision is the treatment of choice for BC. ${ }^{1}$ The radical excision with wider margins is required in case a malignancy is suspected. On histopathology, the presence of ovarian stroma in malignant lesions is the only differentiating factor between benign and malignant cysts. ${ }^{6}$ Three year survival rate of $66 \%$ is reported in malignancy after liver resections. ${ }^{14}$ Adjuvant chemotherapy in case of malignant lesions has been used on trial basis without any definitive protocol. ${ }^{6}$

In conclusion, $\mathrm{BC}$ are rare hepatic SOL. The hydatid cyst and liver abscess may closely resemble BC in endemic areas. Every SOL in liver should prompt early investigation. Liver resection offers the best cure in patients of cystadenoma and cystadenocarcinomas.

\section{ORCID}

Krishna Ramavath: https://orcid.org/0000-0002-1670-3902 Lileswar Kaman: https://orcid.org/0000-0003-1837-2614

Ashish Gupta: https://orcid.org/0000-0001-7413-267X

Abhinav Singh: https://orcid.org/0000-0002-7959-1696

Ashim Das: https://orcid.org/0000-0002-2031-7000

\section{AUTHOR CONTRIBUTIONS}

Conceptualization: LK. Data curation: LK, KR, AG. Formal Analysis: LK, KR, AS, AD. Funding: NA. Methodology: LK, KR, AS. Project Administration: LK. Visualization: LK, AD. Writing- Original draft: LK, AG. Writing - Review and editing: LK.

\section{REFERENCES}

1. Lantinga MA, Gevers TJ, Drenth JP. Evaluation of hepatic cystic lesions. World J Gastroenterol 2013;19:3543-3554.

2. Choi BY, Nguyen MH. The diagnosis and management of benign hepatic tumors. J Clin Gastroenterol 2005;39:401-412.

3. Bacher H, Cerwenka H, Werkgartner G, El-Shabrawi A, Höss G, Preidler K, et al. Primary biliary cystadenocarcinoma perforating the duodenum and left intrahepatic biliary tree--mimicking a hydatid cyst. Liver 1999;19:39-41.

4. Del Poggio P, Buonocore M. Cystic tumors of the liver: a practical approach. World J Gastroenterol 2008;14:3616-3620.

5. Frick MP, Feinberg SB. Biliary cystadenoma. AJR Am J Roentgenol 1982;139:393-395.

6. Simo KA, Mckillop IH, Ahrens WA, Martinie JB, Iannitti DA, Sindram D. Invasive biliary mucinous cystic neoplasm: a review. HPB (Oxford) 2012;14:725-740.

7. Williams DM, Vitellas KM, Sheafor D. Biliary cystadenocarcinoma: seven year follow-up and the role of MRI and MRCP. Magn Reson Imaging 2001;19:1203-1208.

8. Korobkin M, Stephens DH, Lee JK, Stanley RJ, Fishman EK, Francis IR, et al. Biliary cystadenoma and cystadenocarcinoma: CT and sonographic findings. AJR Am J Roentgenol 1989;153: 507-511.

9. Scherer K, Gupta N, Caine WP, Panda M. Differential diagnosis and management of a recurrent hepatic cyst: a case report and review of literature. J Gen Intern Med 2009;24:1161-1165.

10. Koffron A, Rao S, Ferrario M, Abecassis M. Intrahepatic biliary cystadenoma: role of cyst fluid analysis and surgical management in the laparoscopic era. Surgery 2004;136:926-936. 
11. van Roekel V, Marx WJ, Baskin W, Greenlaw RL. Cystadenoma of the liver. J Clin Gastroenterol 1982;4:167-172.

12. Manouras A, Markogiannakis H, Lagoudianakis E, Katergiannakis V. Biliary cystadenoma with mesenchymal stroma: report of a case and review of the literature. World J Gastroenterol 2006; 12:6062-6069.

13. Erdogan D, Busch OR, Rauws EA, van Delden OM, Gouma DJ, van-Gulik TM. Obstructive jaundice due to hepatobiliary cystadenoma or cystadenocarcinoma. World J Gastroenterol 2006; 12:5735-5738

14. Läuffer JM, Baer HU, Maurer CA, Stoupis C, Zimmerman A,
Büchler MW. Biliary cystadenocarcinoma of the liver: the need for complete resection. Eur J Cancer 1998;34:1845-1851.

15. Gupta A, Pattnaik B, Das A, Kaman L. Von Meyenburg complex and complete ductal plate malformation along with Klatskin tumour: a rare association. BMJ Case Rep 2016;2016:10.1136/bcr2016-215220.

16. Kele PG, van der Jagt EJ. Diffusion weighted imaging in the liver. World J Gastroenterol 2010;16:1567-1576.

17. Takanami K, Kaneta T, Yamada S, Takahashi S. F-18 FDG PET/CT scan in biliary cystadenocarcinoma. Clin Nucl Med 2009;34:470-472. 